\title{
The Closed Graph Theorem and the Space of Henstock-Kurzweil Integrable Functions with the Alexiewicz Norm
}

\author{
Luis Ángel Gutiérrez Méndez, Juan Alberto Escamilla Reyna, \\ Maria Guadalupe Raggi Cárdenas, and Juan Francisco Estrada García
}

\begin{abstract}
Facultad de Ciencias Físico Matemáticas, Benemérita Universidad Autónoma de Puebla, Avenida San Claudio y 18 Sur, Colonia San Manuel, 72570 Puebla, PUE, Mexico
\end{abstract}

Correspondence should be addressed to Luis Ángel Gutiérrez Méndez; gutierrezmendezluisangel@yahoo.com.mx

Received 5 June 2012; Accepted 25 December 2012

Academic Editor: Martin Schechter

Copyright (C) 2013 Luis Ángel Gutiérrez Méndez et al. This is an open access article distributed under the Creative Commons Attribution License, which permits unrestricted use, distribution, and reproduction in any medium, provided the original work is properly cited.

We prove that the cardinality of the space $\mathscr{H} \mathscr{K}([a, b])$ is equal to the cardinality of real numbers. Based on this fact we show that there exists a norm on $\mathscr{H} \mathscr{K}([a, b])$ under which it is a Banach space. Therefore if we equip $\mathscr{H} \mathscr{K}([a, b])$ with the Alexiewicz topology then $\mathscr{H} \mathscr{K}([a, b])$ is not K-Suslin, neither infra- $(u)$ nor a webbed space.

\section{Introduction}

Let $[a, b]$ be a compact interval in $\mathbb{R}$. In the vector space of Henstock-Kurzweil integrable functions on $[a, b]$ with values in $\mathbb{R}$ the Alexiewicz seminorm is defined as

$$
\|f\|_{A}=\sup _{a \leq r \leq b}\left|\int_{a}^{r} f\right| .
$$

The corresponding normed space is built using the quotient space determined by the relation $f \sim g$ if and only if $f=g$, except in a set of Lebesgue measure zero or, equivalently, if they have the same indefinite integral. This normed space will be denoted by $\left(\mathscr{H} \mathscr{K}([a, b]),\|\cdot\|_{A}\right)$ and its elements as $f$ instead of $[f]$.

It is a known fact that the space $\left(\mathscr{H} \mathscr{K}([a, b]),\|\cdot\|_{A}\right)$ is not complete nor of the second category [1]. However, the space $\left(\mathscr{H} \mathscr{K}([a, b]),\|\cdot\|_{A}\right)$ has "nice" properties, from the point of view of functional analysis, since it is an ultrabornological space [2].

Höning [3], using a version of the Closed Graph Theorem, proved that there is no natural Banach norm on the space $\mathscr{H} \mathscr{K}([a, b])$; even, for functions with values in a Banach space. Also, he observed that the same proof applies to prove that there is no natural Fréchet topology on the space $\mathscr{H} \mathscr{K}([a, b])$.
Afterwards, using the same technique used by Höning, Merino improves Höning's results in the sense that those results arise as corollaries of the following theorem.

Theorem 1 (see [2]). There exists no ultrabornological, infra(u), natural, and complete topology on $\mathscr{H} \mathscr{K}([a, b])$.

On the basis of Corollary 7 we prove the following result: there exists a norm on $\mathscr{H} \mathscr{K}([a, b])$ under which it is a Banach space; see Proposition 9. Then, based on this fact and using the same techniques employed by Höning and Merino, we prove that the space $\left(\mathscr{H} \mathscr{K}([a, b]),\|\cdot\|_{A}\right)$ does not have certain properties and we give an answer to two problems posed by Merino in [2, page 111]; namely, we will prove, in particular, that the space $\mathscr{H} \mathscr{K}([a, b])$ with the Alexiewicz topology is neither infra- $(u)$ nor a webbed space.

\section{Notation and Preliminaries}

For the reader's convenience, we restate some concepts about topological vector spaces that will be used later in this paper which can be found particularly in [4-6].

Definition 2. Let $X$ and $Y$ be topological spaces and let $f: X \rightarrow Y$ be a function. The graph of $f$ is defined by $\{(x, f(x)) \mid x \in X\}$. 
It is said that $f$ has closed graph if its graph is a closed subset of $X \times Y$ with the product topology.

It is a known fact that if $f: X \rightarrow Y$ is a continuous function between topological spaces, where $Y$ is a Hausdorff space, then $f$ has closed graph; however, the converse is not true in general. Precisely, the versions of the Closed Graph Theorem establish under what conditions the converse is fulfilled. Moreover, the importance of these theorems is that in certain contexts it is easier to prove that a function has closed graph than to prove that it is continuous; Propositions 10,12 , and 14 are examples of this fact.

Definition 3. A topological vector space $X$ is locally convex if $X$ has a local base of convex neighborhoods of 0 .

Definition 4. Let $X$ be a locally convex space. Then $X$ is

(i) barrelled if every linear mapping with closed graph from $X$ into a Banach space $Y$ is continuous;

(ii) infra- $(u)$ if every linear mapping with closed graph from a Banach space $Y$ into $X$ is continuous;

(iii) infra-(s) if every linear mapping with closed graph from a barrelled space $Y$ into $X$ is continuous.

The above concepts have other equivalent characterizations. However, the characterizations that appear in the preceding definition are the best for the goals of this paper.

Definition 5. Let $X$ be a locally convex space. It is said that $X$ is a

(i) webbed space or De Wilde space if it admits a $\mathscr{C}$-web;

(ii) convex-Suslin space if it admits a compacting web.

Note. The definitions of $\mathscr{C}$-web and compacting web are a bit too involved to be here. To see these definitions we refer the reader to [5].

Although webbed spaces are a subclass of infra- $(u)$ spaces, the webbed spaces have more stability properties than infra- $(u)$ spaces in the sense that every sequentially closed subspace and every quotient of a webbed space are webbed spaces. Also the inductive and projective limits and the direct sums and direct products of a countable number of webbed spaces are webbed spaces. Furthermore, if $X$ is a webbed space, then it also will be with a weaker topology. In the other direction, if $X$ is a webbed space, it also will be with the associated ultrabornological topology.

Some stability properties that enjoy the convex-Suslin spaces are the following: the countable product of convexSuslin spaces is convex-Suslin and every closed subspace of a convex-Suslin space is a convex-Suslin space. Moreover, these spaces play a version of Closed Graph Theorem as set forth below.

Theorem 6 (see [6]). Let $Y$ be a locally convex Baire space and let $X$ be a convex-Suslin space. If $f$ is a linear mapping from $Y$ into $X$ with closed graph, then $f$ is continuous.
Now, we establish the notations that we will use throughout this paper.

(i) Let $X$ be a vector space.

(a) All vector spaces that appear throughout this paper are considered over the field of the real or complex numbers.

(b) The cardinality of $X$ will be denoted by $\operatorname{card}(X)$.

(c) The cardinality of a Hamel base or algebraic base is denoted by $\operatorname{dim}(X)$. It is a known fact that every Hamel base in a vector space has the same cardinality.

(ii) The symbols $\aleph_{0}, c$ and $c^{\aleph_{0}}$ will denote the cardinality of the natural numbers, $\mathbb{N}$, of the real numbers, $\mathbb{R}$, and of the set $\{f: \mathbb{N} \rightarrow \mathbb{R} \mid f$ is a function $\}$, respectively.

(iii) We will denote the set of continuous functions with real values whose domain is the compact interval $[a, b]$ by $(\mathscr{C}[a, b], \mathbb{R})$.

(iv) $L^{1}[a, b]$ will denote the space of equivalence classes of the Lebesgue integrable functions with its usual norm. It is a known fact that $L^{1}[a, b] \subset \mathscr{H} \mathscr{K}([a, b])$ and $\|f\|_{A} \leq\|f\|_{1}$, for all $f \in L^{1}[a, b]$.

The following result establishes necessary and sufficient conditions to determine when a vector space has a norm under which it is a Banach space. This result is the foundation of the goals of this paper.

Corollary 7 (see [7]). Let $X$ be a vector space. Then, $X$ is a Banach space under some norm if and only if $\operatorname{dim}(X)<\aleph_{0}$ or $\operatorname{dim}(X)^{\aleph_{0}}=\operatorname{dim}(X)$.

\section{Principal Results}

Lemma 8. The cardinality of the space $\mathscr{H} \mathscr{K}([a, b])$ is equal to the cardinality of real numbers.

Proof. Since the application $G: \mathscr{H} \mathscr{K}([a, b]) \rightarrow(\mathscr{C}[a, b], \mathbb{R})$ defined by $G(f)=F$, where $F$ is the indefinite integral associated to $f$, is injective, it holds that $\operatorname{card}(\mathscr{H} \mathscr{K}([a, b])) \leq$ $\operatorname{card}((\mathscr{C}[a, b], \mathbb{R}))=c$.

On the other hand, as the constant functions are Henstock-Kurzweil integrables, it follows that

$$
\begin{aligned}
c & =\operatorname{card}(\{g:[a, b] \longrightarrow \mathbb{R} \mid g \text { is a constant function }\}) \\
& \leq \operatorname{card}(\mathscr{H} \mathscr{K}([a, b])) .
\end{aligned}
$$

Therefore, $\operatorname{card}(\mathscr{H} \mathscr{K}([a, b]))=c$.

Proposition 9. There exists a norm on $\mathscr{H} \mathscr{K}([a, b])$ under which it is a Banach space.

Proof. It is a known fact that $L^{1}[a, b]$ is a Banach space of infinite dimension, which implies that $c \leq \operatorname{dim}\left(L^{1}[a, b]\right)$. 
Then, as $L^{1}[a, b] \subset \mathscr{H} \mathscr{K}([a, b])$ it holds that $\operatorname{dim}\left(L^{1}[a, b]\right) \leq$ $\operatorname{dim}(\mathscr{H} \mathscr{K}([a, b])) \leq \operatorname{card}(\mathscr{H} \mathscr{K}([a, b]))$. Therefore, by Lemma 8, Corollary 7 and the known fact that $c^{\aleph_{0}}=c$, we obtain the desired conclusion.

Hereafter, the Alexiewicz topology and the topology induced by the norm of Proposition 9 will be denoted as $\tau_{A}$ and $\tau_{\|\cdot\|}$, respectively.

Proposition 10. The topology $\tau_{\|\cdot\|}$ on $\mathscr{H} \mathscr{K}([a, b])$ is smaller than $\tau_{A}$.

Proof. Let $\|\cdot\|$ be the norm ensured by Proposition 9. By [3], $\left(\mathscr{H} \mathscr{K}([a, b]), \tau_{A}\right)$ is barrelled and, therefore, the identity function

$$
\mathscr{I}:\left(\mathscr{H} \mathscr{K}([a, b]), \tau_{A}\right) \longrightarrow\left(\mathscr{H} \mathscr{K}([a, b]), \tau_{\|\cdot\|}\right),
$$

which has a closed graph, is continuous; hence $\tau_{\|\cdot\|} \subseteq \tau_{A}$. In addition, since that $\tau_{A}$ is not complete [3], it holds that $\tau_{\|\cdot\|} \subset$ $\tau_{A}$.

Although $\left(\mathscr{H} \mathscr{K}([a, b]), \tau_{\|\cdot\|}\right)$ is a Banach space, according to [3] the topology $\tau_{\|\cdot\|}$ is not natural in the sense of the following definition.

Definition 11. A vector topology $\tau$ on $\mathscr{H} \mathscr{K}([a, b])$ is natural if every sequence $f_{n}$ in $\mathscr{H} \mathscr{K}([a, b])$ such that $f_{n} \stackrel{\tau}{\rightarrow} 0$ implies that $\int_{a}^{x} f_{n} \rightarrow 0$, for every $x \in[a, b]$.

Now, we shall prove that the space $\left(\mathscr{H} \mathscr{K}([a, b]), \tau_{A}\right)$ is not infra- $(u)$ which follows from the following general result.

Proposition 12. Let $(X, \tau)$ be a barrelled space that is not complete. If there exists a norm on $X$ under which it is a Banach space, then $X$ is not an infra-(u) space.

Proof. Let $\|\cdot\|$ be a norm in $X$ such that $(X,\|\cdot\|)$ is a Banach space and suppose that $(X, \tau)$ is an infra- $(u)$ space. Since $(X, \tau)$ is a barrelled space, it holds that the identity function

$$
\mathscr{I}:(X, \tau) \longrightarrow(X,\|\cdot\|)
$$

which has a closed graph, is continuous; hence $\tau_{\|\cdot\|} \subseteq \tau$. Then, as $(X, \tau)$ is infra- $(u)$ and $(X,\|\cdot\|)$ is a Banach space, it follows that

$$
\mathscr{I}^{-1}:(X,\|\cdot\|) \longrightarrow(X, \tau)
$$

is continuous; hence $\tau \subseteq \tau_{\|\cdot\| \cdot}$.

Therefore $\tau=\tau_{\|\cdot\|}$ is a contradiction because $\tau$ is not complete.

Corollary 13. The space $\left(\mathscr{H} \mathscr{K}([a, b]), \tau_{A}\right)$ is not infra- $(u)$.

Proof. On the basis of Proposition 9 there exists a norm on $\mathscr{H} \mathscr{K}([a, b])$ under which it is a Banach space. Furthermore, according to [3] the space $\left(\mathscr{H} \mathscr{K}([a, b]), \tau_{A}\right)$ is barrelled but not complete. Therefore, it follows from Proposition 12 that $\left(\mathscr{H} \mathscr{K}([a, b]), \tau_{A}\right)$ is not infra- $(u)$.
On the basis of Corollary 13 we see that $\left(\mathscr{H} \mathscr{K}([a, b]), \tau_{A}\right)$ is not a webbed space. Moreover, the same result implies that $\left(\mathscr{H} \mathscr{K}([a, b]), \tau_{A}\right)$ is not an infra- $(s)$ space, a fact that Merino has already proved in [2].

On the other hand, employing the same technique that we have been using entails the following result.

Proposition 14. The space $\left(\mathscr{H} \mathscr{K}([a, b]), \tau_{A}\right)$ is not a convexSuslin space.

Proof. Suppose that $\left(\mathscr{H} \mathscr{K}([a, b]), \tau_{A}\right)$ is a convexSuslin space. On the basis of Proposition 9 we see that $\left(\mathscr{H} \mathscr{K}([a, b]), \tau_{\|\cdot\|}\right)$ is a locally convex Baire space; therefore, according to Theorem 6 the identity function

$$
\mathscr{I}:\left(\mathscr{H} \mathscr{K}([a, b]), \tau_{\|\cdot\|}\right) \longrightarrow\left(\mathscr{H} \mathscr{K}([a, b]), \tau_{A}\right),
$$

which has a closed graph, is continuous, contradicting Proposition 10.

Since K-Suslin spaces are a subclass from convex-Suslin spaces, [6], we can see, on the basis of Proposition 14, that $\left(\mathscr{H} \mathscr{K}([a, b]), \tau_{A}\right)$ is not a $\mathrm{K}-$ Suslin space.

Although apparently the topology $\tau_{\|\cdot\|}$ is not directly related with the Henstock-Kurzweil integral, this topology allowed us to demonstrate some properties that the Alexiewicz topology does not have, which is a topology related to the Henstock-Kurzweil integral since it is a natural topology.

\section{Comments}

(i) There are other types of spaces which enjoy certain versions of Closed Graph Theorem, for example, the spaces: Suslin, quasi-Suslin, [8], and A-complete, [9]; Ptak and infra-Pták [10]; strongly first-countable convergence vector space [11]; and so forth. Then, applying the same technique that we have used in this paper it can be shown that the space $\left(\mathscr{H} \mathscr{K}([a, b]), \tau_{A}\right)$ does not belong to any class of the spaces above.

(ii) As another application of the technique which has been used in this paper and reiterating the known fact that $L^{1}[a, b] \subset \mathscr{H} \mathscr{K}([a, b])$ and $\|f\|_{A} \leq$ $\|f\|_{1}$, for all $f \in L^{1}[a, b]$, it can be shown that $\left(L^{1}[a, b],\|\cdot\|_{A}\right)$ is not a barrelled space and therefore not an ultrabornological space.

\section{Acknowledgment}

This paper is supported by the Committee of Differential Equations and Mathematical Modeling from FCFM-BUAP through Dr. José Jacobo Oliveros Oliveros.

\section{References}

[1] C. Swartz, Introduction to Gauge Integrals, World Scientific, Singapore, 2001.

[2] J. L. G. Merino, Integraciones de Denjoy de funciones con valores en espacios de Banach [Ph.D. thesis], Universidad Complutense de Madrid, 1997. 
[3] C. S. Höning, “There is no natural Banach space norm on the space of Kurzweil-Henstock-Denjoy-Perron integrable functions," in Proceedings of the 30th Seminario Brasileiro de Análise, October 1989.

[4] P. Pérez Carreras and J. Bonet, Barrelled Locally Convex Spaces, vol. 131 of North-Holland Mathematics Studies, North-Holland, Amsterdam, The Netherlands, 1987.

[5] G. Köthe, Topological Vector Spaces. II, vol. 237 of Grundlehren der Mathematischen Wissenschaften, Springer, New York, NY, USA, 1979.

[6] J. C. Ferrando, "On convex-Suslin spaces," Acta Mathematica Hungarica, vol. 55, no. 3-4, pp. 201-206, 1990.

[7] A. H. Kruse, "Badly incomplete normed linear spaces," Mathematische Zeitschrift, vol. 83, pp. 314-320, 1964.

[8] M. Nakamura, "On quasi-Souslin space and closed graph theorem," Proceedings of the Japan Academy, vol. 46, pp. 514517, 1970.

[9] Q. D. Zhu and Z. X. Zhao, "A-complete spaces and the closed graph theorem," Acta Mathematica Sinica, vol. 24, no. 6, pp. 833836, 1981.

[10] E. Pap and C. Swartz, "On the closed graph theorems," in Generalized Functions and Convergence, pp. 355-359, World Scientific, Teaneck, NJ, USA, 1990.

[11] R. Beattie and H.-P. Butzmann, "Countability, completeness and the closed graph theorem," in Generalized Functions, Convergence Structures, and Their Applications, pp. 375-381, Plenum Press, New York, NY, USA, 1988. 


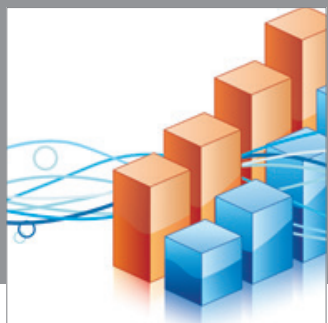

Advances in

Operations Research

mansans

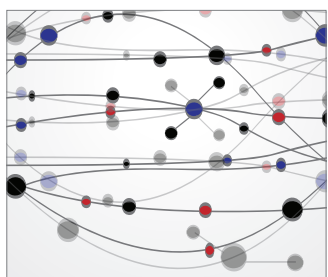

The Scientific World Journal
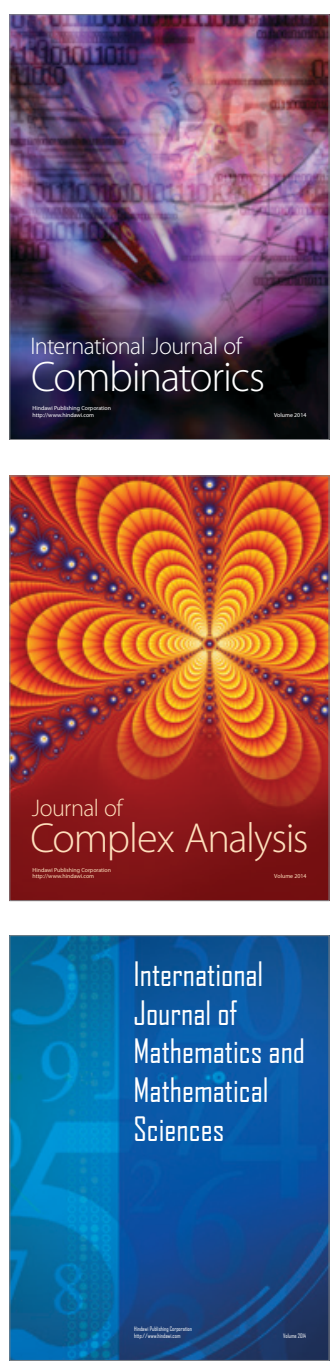
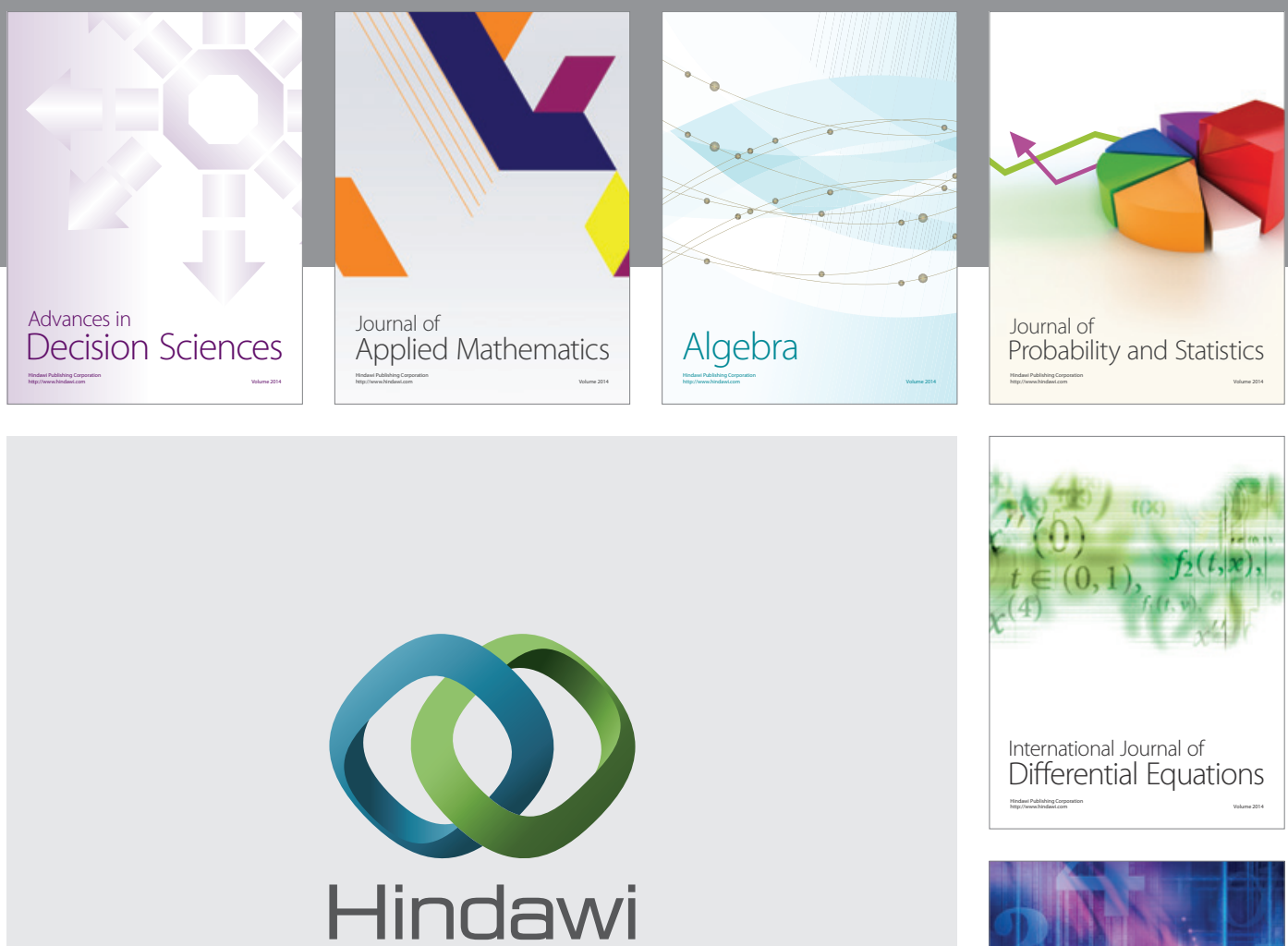

Submit your manuscripts at http://www.hindawi.com
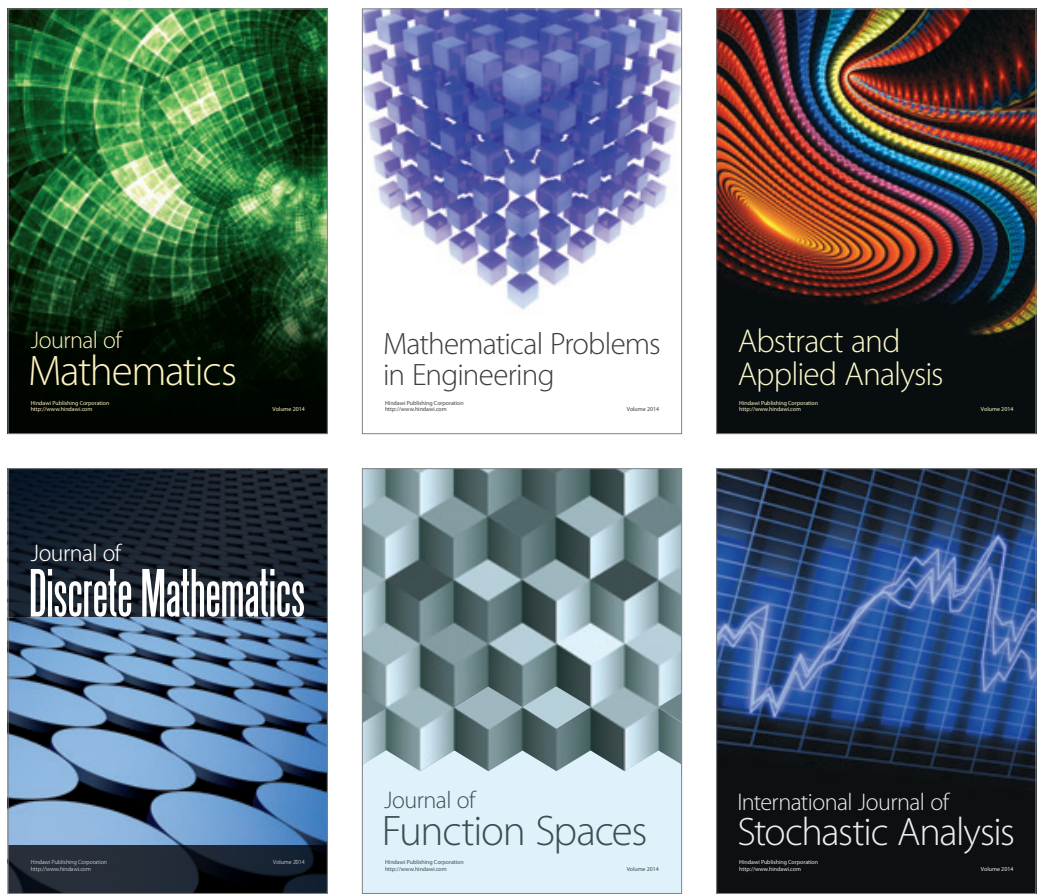

Journal of

Function Spaces

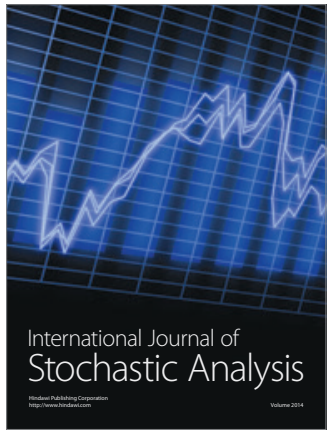

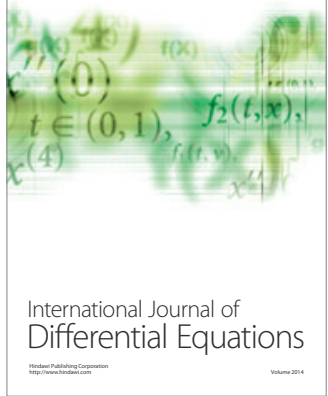
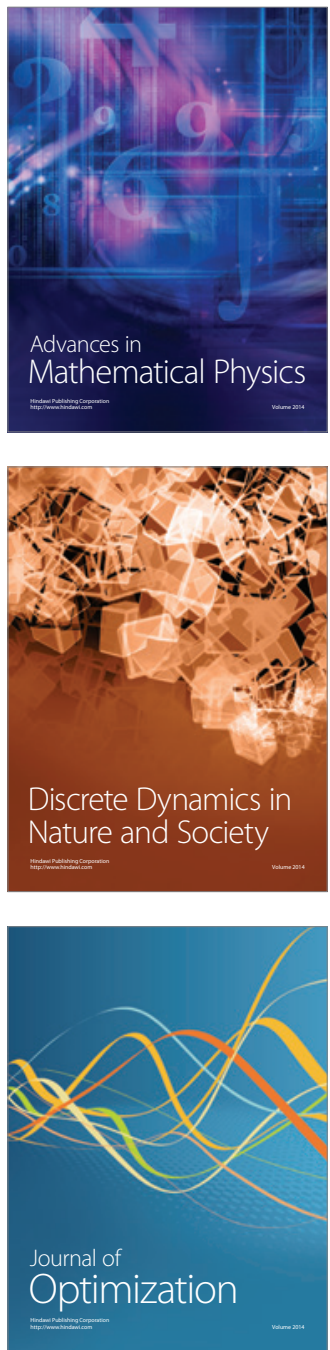